\title{
Estado nutricional e consumo alimentar de pacientes com câncer atendidos em um hospital público de Aracaju-SE
}

\author{
Nutritional status and food consumption of cancer patients treated at a public hospital in Aracaju- \\ SE
Estado nutricional y consumo de alimentos de pacientes oncológicos atendidos en un hospital público de Aracaju-SE

\section{Resumo}

O papel de um profissional nutricionista em setores oncológicos é contribuir na recuperação e na manutenção do estado nutricional do paciente, o aconselhamento e a intervenção nutricional antes e durante o tratamento são fatores indispensáveis nesse processo. O objetivo do trabalho foi avaliar o estado nutricional e adequação da ingestão alimentar de pacientes com Câncer de um hospital público de Aracaju-SE. A pesquisa contou com pacientes oncológicos, adultos e idosos, de ambos os sexos. Foram coletadas as medidas de peso, estatura, circunferência do braço, prega cutânea tricipital, além disso foi coletado o recordatório alimentar de 24 horas. Do total de indivíduos avaliados $(\mathrm{n}=167)$ as mulheres foram maioria 74,3\% ( $n=124)$ e apresentaram maiores casos de alterações no estado nutricional. O câncer de mama aparece como o de maior número de casos 35,3\% (n=59), dos tratamentos utilizados a quimioterapia foi a de maior uso 53,9\% (n=90). Com relação ao consumo alimentar, potássio, cálcio, ferro (51-70), magnésio, sódio (31-50) e as vitaminas A, D, E, B1, B6 (51-70) e B9 estavam abaixo da recomendação adequada de acordo com as Dietary Reference Intakes (DRIs): Encontraram-se resultados significativos para maior perda de peso em mulheres além de uma inadequação dos micronutrientes, sendo necessário o acompanhamento nutricional para estabilizar ou minimizar essas alterações.

Palavras-chave: Estado nutricional; Antropometria; Oncologia; Consumo de alimentos.

\section{Abstract}

The role of a professional nutritionist in oncology sectors is to contribute to the recovery and maintenance of the patient's nutritional status, nutritional counseling and intervention before and during treatment are indispensable factors in this process. The objective of this study was to evaluate the nutritional status and adequacy of food intake of patients 
with cancer at a public hospital in Aracaju SE. The research included cancer patients, adults and elderly, of both sexes. Measures of weight, height, arm circumference, triceps skinfold were collected, in addition to the 24-hour dietary recall. Of the total number of individuals evaluated, women were the majority $74.3 \%(n=124)$ and had higher cases of alterations in their nutritional status. Breast cancer appears as the largest number of cases, 35.3\% $(n=59)$, of the treatments used in chemotherapy for the greatest use, 53.9\% ( $\mathrm{n}=90)$. Regarding food consumption, potassium, calcium, iron (51-70), magnesium, sodium (31-50) and vitamins A, D, E, B1, B6 (51-70) and B9 were below the appropriate recommendation for according to the Dietary Reference Intakes (DRIs).: Results were found for greater weight loss in women in addition to an inadequacy of micronutrients, requiring nutritional monitoring to stabilize or minimize these changes.

Keywords: Nutritional status; Anthropometry; Medical oncology; Food consumption.

\section{Resumen}

El papel de un nutricionista profesional en los sectores de la oncología es contribuir a la recuperación y mantenimiento del estado nutricional del paciente, el asesoramiento y la intervención nutricional antes y durante el tratamiento son factores fundamentales en este proceso. El objetivo de este estudio fue evaluar el estado nutricional y la adecuación de la ingesta alimentaria de pacientes oncológicos en un hospital público de Aracaju SE. La investigación incluyó a pacientes con cáncer, adultos y ancianos, de ambos sexos. Se recopilaron medidas de peso, altura, circunferencia del brazo, pliegue cutáneo del tríceps, además del recordatorio dietético de 24 horas. Del total de individuos evaluados, las mujeres constituyeron la mayoría $74,3 \%(n=124)$ y presentaron mayores casos de alteraciones en su estado nutricional. El cáncer de mama aparece como el que presenta mayor número de casos 35,3\% ( $\mathrm{n}=59)$, de los tratamientos utilizados, la quimioterapia fue el más utilizado $53,9 \%(\mathrm{n}=90)$. En cuanto a la ingesta de alimentos, el potasio, calcio, hierro $(51-$ 70), magnesio, sodio (31-50) y vitaminas A, D, E, B1, B6 (51-70) y B9 estuvieron por debajo de la recomendación adecuada de según la Ingestas Dietéticas de Referencia (DRI): Se encontraron resultados significativos para una mayor pérdida de peso en las mujeres, además de una insuficiencia de micronutrientes, requiriendo un seguimiento nutricional para estabilizar o minimizar estos cambios.

Palabras clave: Estado nutricional; Antropometría; Oncología médica; Consumo de alimentos.

\section{Introdução}

O câncer é uma doença crônica de origem multifatorial, na qual há crescimento irregular e descontrolado de células que são capazes de se multiplicarem e se infiltrar em diversos órgãos e tecidos. Essa patologia é apontada como um problema de saúde pública tanto em países desenvolvidos quanto nos subdesenvolvidos (Silva et al., 2012). Cogitam-se para o Brasil, 625 mil casos novos de câncer no biênio 2020-2022 (INCA, 2020).

Dentre os tratamentos utilizados para o combate ao câncer, a quimioterapia é a mais usada (90\% dos casos), a qual se utilizam 300 tipos de drogas que atuam impedindo o crescimento das células anormais. Entretanto, essa terapia resulta em dados laboratoriais alterados e vários efeitos colaterais (Gozzo et al., 2015). Kanda et al. (2014) afirma que os eventos adversos mais frequentes são apatia e alopecia, causados pela baixa imunidade, e inapetência, náuseas, êmeses, entre outros, e que causam o aumento da morbimortalidade no paciente.

A presença do tumor afeta as necessidades nutricionais do organismo e a desnutrição é muito frequente. As alterações metabólicas inerentes ao crescimento dos tumores, como: aumento da lipólise, da utilização de glicose, da produção de marcadores inflamatórios, aumento do gasto energético e inapetência, liberação de substâncias catabólicas etc. Além do tratamento antineoplásico, contribuem para a depleção nutricional e a redução da qualidade de vida do paciente (Port, 2012).

Durante o processo de tratamento, o consumo alimentar sofre alterações por fatores psicológicos, emocionais e sintomas provenientes da doença. Essas interferências resultam em uma significativa diminuição dos alimentos ingeridos, podendo levar ao comprometimento do estado nutricional (Poltronieri \& Tusset, 2016).

A alimentação saudável ajuda na prevenção ou mesmo atrasa o seu surgimento e, principalmente, durante o tratamento da doença em que a maioria dos quimioterápicos ocasiona efeitos indesejáveis. Alimentos como frutas e hortaliças têm assumido posição de destaque nos estudos que envolvem a prevenção do câncer. Esses alimentos devem fazer parte da alimentação diária, por serem antioxidantes, devido a ação protetora efetiva contra os processos oxidativos que ocorrem no organismo e serem capazes de estabilizar ou desativar os radicais livres do organismo (Azevedo \& Dal Bosco, 2011). 
A avaliação antropométrica constitui um dos parâmetros para avaliação nutricional deste paciente, uma vez que possibilita identificar o risco nutricional do mesmo. Ressalta-se também a importância da relação entre a alimentação e o câncer no estado nutricional, haja visto que este pode sofrer mudanças decorrente da própria doença, do aumento das necessidades nutricionais, além dos efeitos colaterais advindos do tratamento oncológico, contribuindo assim para o desenvolvimento da desnutrição (Souza et al., 2017).

$\mathrm{O}$ aconselhamento e a intervenção nutricional antes e durante o tratamento acaba sendo um fator indispensável quando se trata da recuperação do estado nutricional de pacientes oncológicos justamente pelo fato de que esses pacientes necessitam de uma oferta calórica ideal e individualizada, assim como à ingestão de nutrientes e líquidos para a sua evolução clínica (Pootz et al., 2020).

O presente trabalho teve a intenção de avaliar o estado nutricional e adequação da ingestão alimentar de pacientes com Câncer de hospital público de Aracaju-SE.

\section{Metodologia}

O presente estudo foi realizado no ambulatório de oncologia no setor de nutrição de um hospital público de Aracaju/SE. O critério utilizado para a seleção da amostra foi ter o diagnóstico de câncer, independente do grau de estadiamento e tipo de tratamento realizado, sem restrição de idade e sexo. Foram excluídos do estudo aqueles pacientes em cuidados paliativos.

O estudo tem caráter transversal, descritivo, realizado por conveniência, com a participação de pacientes oncológicos, adultos e idosos, de ambos os sexos, que foram atendidos no ambulatório de oncologia no setor de nutrição de um hospital público de Aracaju/SE. Todos os participantes foram incluídos mediante assinatura do Termo de Consentimento Livre e Esclarecidos (TCLE). Foram excluídos da pesquisa os pacientes que estavam em terapia nutricional parenteral. Através do questionário, foram registradas informações demográficas e alimentares e, observadas sinais e sintomas durante a internação.

Para avaliação do estado nutricional, foram utilizados dados antropométricos. O índice de massa corporal foi calculado a partir da razão entre o peso $(\mathrm{Kg})$ e a estatura $(\mathrm{m})$ ao quadrado. Para classificação do estado nutricional pelo IMC foram utilizados os pontos de corte propostos pela World Health Organization (1998) para os adultos e Lipschitz (1994) para os idosos. A circunferência da cintura (CC), com o objetivo de avaliar a adiposidade abdominal em função da sua associação com risco de doenças cardiovasculares (DCV), foi medida com fita métrica inelástica no ponto médio entre o último arco costal e a crista ilíaca e classificada de acordo com a World Health Organization (1998).

A antropometria foi realizada por meio das medidas de peso e estatura estimada utilizando as metodologias de Chumlea et al. (1988) e Chumlea et al. (1994) para o cálculo do índice de massa corporal (IMC). A CB foi medida com fita métrica inelástica no ponto médio entre o acrômio e o olécrano, com o braço relaxado; a PCT com adipômetro científico do tipo Lange® (0 a $60 \mathrm{~mm} \times 1 \mathrm{~mm}$ ), na parte posterior do braço, na altura do ponto médio medido para a CB. Ambas as medidas foram classificadas por percentis de acordo com Frisancho para adultos e NHANES III para os idosos (Frisancho, 1990; Kuczmarski et al., 2000).

Para quantificar a ingestão de nutrientes, o consumo alimentar relatado pelos pacientes foi avaliado por meio da aplicação de 1 recordatório alimentar de 24 horas (R24h) e os alimentos foram registrados na forma de medidas caseiras. Os cálculos referentes a ingestão de energia, macronutrientes, micronutrientes, colesterol e fibras alimentares foram realizados através do programa Dietbox. Quanto a avaliação da adequação dos macronutrientes e micronutrientes, foi utilizado a tabela do Dietary Reference Intakes (DRI) (Padovani et al., 2006).

A pesquisa foi aprovada pelo Comitê de Ética em Pesquisa sob o CAAE: 51765015.4.0000.5371 e seguindo as normas estabelecidas na Resolução 466/2012 (Brasil, 2012).

As variáveis discretas foram analisadas a partir de sua frequência nos pontos de corte específicos (gênero, IMC, CC). 
As variáveis contínuas foram apresentadas em média e desvio padrão ( $\mathrm{X} \pm \mathrm{DP}$ ). Foi utilizado o teste de associação do quiquadrado com nível de significância de $\mathrm{p} \leq 0,05$. A avaliação dietética foi analisada no software de nutrição e analisada com base nas Dietary Reference Intakes (DRIs).

\section{Resultados}

A amostra foi composta por 167 pacientes. Com relação as características dos pacientes 74,3\% $(\mathrm{n}=124)$ dos pacientes eram do sexo feminino, sendo 68,9\% $(n=115)$ adultos. Foi observado também com relação à escolaridade que a maioria dos pacientes apesar de serem adultos, não tinham escolaridade completa, 24,6\% ( $n=41)$ estavam no grupo de tabagismo, sendo o restante dos pacientes ex-tabagistas ou não tabagistas, com relação à dentição, o número de pacientes que não tem ou que faz o uso de prótese chega a 71,2\% $(\mathrm{n}=119)$. Observando a atividade física 66,7\% $(\mathrm{n}=111)$ não praticavam atividades físicas. (Tabela 1).

Tabela 1. Caracterização dos pacientes atendidos no ambulatório de nutrição da oncologia de um hospital público de AracajuSE.

\begin{tabular}{|c|c|c|c|}
\hline & & $\mathbf{N}$ & $\%$ \\
\hline \multirow[t]{2}{*}{ Gênero } & Feminino & 124 & 74,3 \\
\hline & Masculino & 43 & 25,7 \\
\hline \multirow[t]{2}{*}{ Faixa Etária } & Adulto & 115 & 68,9 \\
\hline & Idoso & 52 & 31,1 \\
\hline \multirow[t]{7}{*}{ Escolaridade } & Analfabeto & 53 & 31,7 \\
\hline & Fundamental incompleto & 47 & 28,1 \\
\hline & Fundamental completo & 24 & 17,4 \\
\hline & Médio Incompleto & 4 & 2,4 \\
\hline & Médio completo & 29 & 17,4 \\
\hline & Superior incompleto & 3 & 1,8 \\
\hline & Superior completo & 7 & 4,2 \\
\hline \multirow[t]{3}{*}{ Etilismo } & Sim & 6 & 3,6 \\
\hline & Não & 126 & 75,4 \\
\hline & Ex-etilista & 35 & 21 \\
\hline \multirow[t]{3}{*}{ Tabagismo } & Sim & 41 & 24,6 \\
\hline & Não & 81 & 48,5 \\
\hline & Ex-tabagista & 45 & 26,9 \\
\hline \multirow[t]{3}{*}{ Dentição } & Tem & 48 & 28,7 \\
\hline & Não tem & 53 & 31,7 \\
\hline & Uso de prótese & 66 & 39,5 \\
\hline \multirow[t]{2}{*}{ Atividade Física } & Pratica & 56 & 33,53 \\
\hline & Não Pratica & 111 & 66,47 \\
\hline \multirow[t]{7}{*}{ Tipo de Atividade } & Caminhada & 48 & 28,7 \\
\hline & Bicicleta & 1 & 0,6 \\
\hline & Natação & 2 & 1,2 \\
\hline & Academia & 2 & 1,2 \\
\hline & Futebol & 1 & 0,6 \\
\hline & Pilates & 1 & 0,6 \\
\hline & Artes maciais & 1 & 0,6 \\
\hline
\end{tabular}

Fonte: Autores. 
Em relação ao tipo de tratamento a maioria dos pacientes estavam realizando apenas a quimioterapia, sendo 53,9\% $(\mathrm{n}=$ 90), já os pacientes que estavam fazendo a quimioterapia e a radioterapia chegou aos $24 \%(n=40), 11,4 \%(n=19)$ estavam realizando apenas a radioterapia e o restante dos pacientes ou não tinha iniciado ou não fez tratamento nenhum.

Com relação a comorbidade, a maioria dos pacientes não apresentavam nenhuma das comorbidades, chegando a um percentual de 60,5\% $(n=101) .68,3 \%(n=114)$ dos pacientes apresentaram sintomas durante o tratamento, já nos sintomas após o tratamento observamos uma enorme queda na quantidade dos sintomas causados pelos tratamentos, sendo $77,8 \%(\mathrm{n}=130)$ a porcentagem dos pacientes que não apresentaram nenhum sintoma. O restante apresentou vários sintomas ou apenas um sintoma (Tabela 2).

Tabela 2. Característica de saúde dos pacientes atendidos no ambulatório de nutrição da oncologia de um hospital público de Aracaju-SE.

\begin{tabular}{|c|c|c|c|}
\hline & & $\mathbf{N}$ & $\%$ \\
\hline Sitio & Mama & 59 & 35,3 \\
\hline \multirow{8}{*}{ (Localização do câncer) } & Útero/ovário & 28 & 16,8 \\
\hline & Cabeça e pescoço & 19 & 11,4 \\
\hline & Intestino & 18 & 10,8 \\
\hline & Hematológico & 11 & 6,6 \\
\hline & Próstata & 10 & 6,0 \\
\hline & Estomago & 9 & 5,4 \\
\hline & Fígado & 4 & 2,3 \\
\hline & Outros & 9 & 5,4 \\
\hline \multirow[t]{5}{*}{ Tipo de Tratamento } & Quimioterapia & 90 & 53,9 \\
\hline & Quimioterapia e Radioterapia & 40 & 24 \\
\hline & Radioterapia & 19 & 11,4 \\
\hline & Não iniciou & 8 & 4,7 \\
\hline & Não fez tratamento & 10 & 6,0 \\
\hline \multirow{6}{*}{ Presença de comorbidade } & Diabetes & 4 & 2,3 \\
\hline & Hipertensão arterial sistêmica & 20 & 12 \\
\hline & Dislipidemia & 6 & 3,6 \\
\hline & Cardiopata & 6 & 3,6 \\
\hline & Mais de uma comorbidade & 30 & 18 \\
\hline & Não apresenta comorbidade & 101 & 60,5 \\
\hline \multirow[t]{3}{*}{ Sintomas durante o tratamento } & Apresentou vários sintomas & 114 & 68,3 \\
\hline & Apresentou um dos sintomas & 10 & 6 \\
\hline & Não apresentou sintomas & 43 & 25,7 \\
\hline \multirow[t]{3}{*}{ Sintomas após o tratamento } & Apresentou vários sintomas & 25 & 15 \\
\hline & Apresentou pelo menos um sintoma & 12 & 7,2 \\
\hline & Não apresentou sintomas & 130 & 77,8 \\
\hline
\end{tabular}

Fonte: Autores.

Entre os sintomas comumente associados ao tratamento oncológico, foram observados que os pacientes apresentavam em maior quantidade esses sintomas em conjunto durante e depois do tratamento 68,3\% ( $\mathrm{n}=114$ ) e 15\% ( $\mathrm{n}=25$ ), quando observados individualmente a xerostomia foi o que mais foi relatado durante o tratamento ambulatorial 2,5\% (n=4), já após o tratamento o sinal mais contemplado foi a insônia com 2,4\% $(\mathrm{n}=4)$. Um número muito elevado desses pacientes relatou não apresentar quais desses sintomas sendo durante o tratamento 25,7\% (n=43) e após o tratamento 77,8\% (n=130) $($ Tabela 3). 
Tabela 3. Presença de sintomas, relacionados ao tratamento oncológico, apresentados pelos pacientes atendidos no ambulatório de nutrição da oncologia de um hospital público de Aracaju-SE durante e após o tratamento.

\begin{tabular}{|c|c|c|c|c|}
\hline & \multicolumn{2}{|c|}{ Durante o tratamento } & \multicolumn{2}{|c|}{ Após o tratamento } \\
\hline & $\mathbf{N}$ & $\%$ & $\mathbf{N}$ & $\%$ \\
\hline Náuseas & 1 & 0,6 & 2 & 1,2 \\
\hline Diarreia & 0 & 0 & 1 & 0,6 \\
\hline Constipação & 1 & 0,6 & 2 & 1,2 \\
\hline Disfagia & 0 & 0 & 1 & 0,6 \\
\hline Mucosite & 0 & 0 & 0 & 0 \\
\hline Xerostomia & 4 & 2,4 & 1 & 0,6 \\
\hline Disgeusia & 0 & 0 & 0 & 0 \\
\hline Inapetência & 1 & 0,6 & 1 & 0,6 \\
\hline Depressão & 1 & 0,6 & 1 & 0,6 \\
\hline Insônia & 1 & 0,6 & 4 & 2,4 \\
\hline Vários desses sintomas & 114 & 68,3 & 25 & 15 \\
\hline
\end{tabular}

Fonte: Autores.

No que se diz a respeito ao estado nutricional tendo em referência ao IMC, 56,3\% (n=94) eram acima do peso, dentre essas classificações as mulheres eram as que mais se encontravam com excesso de peso em comparação aos homens, sendo eles 79 mulheres e 15 homens. A partir das análises da CB, 58,7\% (n=98) estavam desnutridos sendo a desnutrição moderada a mais predominante com 24,5\% ( $\mathrm{n}=41)$ tendo o público feminino como predominante 31 mulheres e 10 homens com desnutrição moderada. Em relação às medidas da DCT predominou a desnutrição 64,6\% (n=108) sendo mais predominante a desnutrição grave com 40,1\% (n=67), tendo as mulheres com dominante com 41 mulheres e 26 homens com desnutrição grave (Tabela 4 e $5)$.

Tabela 4. Estado nutricional dos pacientes atendidos no ambulatório de nutrição da oncologia de um hospital público de Aracaju-SE.

\begin{tabular}{|c|c|c|c|}
\hline & & $\mathbf{N}$ & $\%$ \\
\hline \multirow{3}{*}{ Índice de massa corporal } & Baixo Peso/Magreza & 24 & 14,4 \\
\hline & Eutrofia & 49 & 29,3 \\
\hline & Acima do peso & 94 & 56,3 \\
\hline \multirow[t]{6}{*}{ Circunferência do braço } & Desnutrido grave & 25 & 15 \\
\hline & Desnutrido moderado & 41 & 24,5 \\
\hline & Desnutrido leve & 32 & 19,2 \\
\hline & Eutrófico & 40 & 24 \\
\hline & Sobrepeso & 14 & 8,3 \\
\hline & Obesidade & 15 & 9 \\
\hline \multirow[t]{6}{*}{ Dobra cutânea do tríceps } & Desnutrido grave & 67 & 40,1 \\
\hline & Desnutrido moderado & 15 & 9 \\
\hline & Desnutrido leve & 26 & 15,5 \\
\hline & Eutrófico & 25 & 15 \\
\hline & Sobrepeso & 9 & 5,4 \\
\hline & Obesidade & 25 & 15 \\
\hline \multirow[t]{3}{*}{ Alteração do Peso } & Não mudou & 104 & 62,3 \\
\hline & Ganhou peso & 11 & 6,6 \\
\hline & Perdeu peso & 52 & 32,1 \\
\hline
\end{tabular}

Fonte: Autores. 
Tabela 5. Associação entre estado nutricional e gênero dos pacientes dos pacientes atendidos no ambulatório de nutrição da oncologia de um hospital público de Aracaju-SE.

\begin{tabular}{|c|c|c|c|c|}
\hline & & Feminino & Masculino & $* \mathbf{p}$ \\
\hline \multirow[t]{3}{*}{ Índice de massa corporal } & Baixo peso & 11 & 13 & \multirow{3}{*}{0,000} \\
\hline & Eutrófico & 34 & 15 & \\
\hline & Acima do peso & 79 & 15 & \\
\hline \multirow[t]{6}{*}{ Circunferência do braço } & Desnutrido grave & 12 & 13 & \multirow{6}{*}{0,001} \\
\hline & Desnutrido moderado & 31 & 10 & \\
\hline & Desnutrido leve & 20 & 12 & \\
\hline & Eutrófico & 34 & 6 & \\
\hline & Sobrepeso & 12 & 2 & \\
\hline & Obesidade & 15 & 0 & \\
\hline \multirow[t]{6}{*}{ Dobra cutânea do tríceps } & Desnutrido grave & 41 & 26 & \multirow{6}{*}{0,03} \\
\hline & Desnutrido moderado & 12 & 3 & \\
\hline & Desnutrido leve & 20 & 6 & \\
\hline & Eutrófico & 20 & 5 & \\
\hline & Sobrepeso & 8 & 1 & \\
\hline & Obesidade & 23 & 2 & \\
\hline \multirow[t]{3}{*}{ Alterou peso } & Não mudou de peso & 76 & 28 & \multirow{3}{*}{0,81} \\
\hline & Ganhou peso & 9 & 2 & \\
\hline & Perdeu peso & 39 & 13 & \\
\hline
\end{tabular}

Teste Qui-quadrado com ${ }^{*} \mathrm{p} \leq 0,05$. Fonte: Autores.

Com relação ao consumo alimentar (Tabela 6), foi possível observar que a média de ingestão de potássio, cálcio, ferro (51-70), magnésio, sódio (31-50) e as vitaminas A, D, E, B1, B6 (51-70) e B9 estavam abaixo da recomendação adequada de acordo com as DRIs, enquanto que fósforo, ferro (31-50), selênio e as vitaminas B2, B3, B6 (31-50) e C estavam acima do recomendado. No tocante a ingestão de macronutrientes (Tabela 7), notou-se que estão de acordo com o recomendado pela DRI.

Tabela 6. Média e desvio padrão da ingestão de energia, micronutrientes e porcentagem de adequação em relação às recomendações nutricionais (DRIs), após análise do recordatório alimentar de 24 horas (R24h) de pacientes atendidos no ambulatório de nutrição da oncologia de um hospital público de Aracaju-SE.

\begin{tabular}{|c|c|c|c|c|}
\hline & $\begin{array}{c}\text { Mediana } \\
\text { (Mínimo - Máximo) }\end{array}$ & $\begin{array}{c}\text { Grupo Etário } \\
\text { (Anos) }\end{array}$ & EAR / AI* & $\begin{array}{c}\text { Percentual de } \\
\text { adequação }\end{array}$ \\
\hline Vitamina A (mcg/dia) & $\begin{array}{c}242,5 \\
(14,01-12.617)\end{array}$ & $31-70$ & 500 & $48,7 \%$ \\
\hline Vitamina D (mcg/dia) & $\begin{array}{c}1,9 \\
(0,0-15,92)\end{array}$ & $31-70$ & 10 & $19 \%$ \\
\hline Vitamina E (mg/dia) & $\begin{array}{c}4,54 \\
(0,18-21,13)\end{array}$ & $31-70$ & 12 & $37,8 \%$ \\
\hline Vitamina B1 (mg/dia) & $\begin{array}{c}0,8 \\
(0,10-4,6)\end{array}$ & $31-70$ & 0,9 & $88,8 \%$ \\
\hline Vitamina B2 (mg/dia) & $\begin{array}{c}1,2 \\
(0,17-6,47)\end{array}$ & $31-70$ & 0,9 & $133,3 \%$ \\
\hline Vitamina B3 (mg/dia) & $\begin{array}{c}13,08 \\
(0,58-54,52)\end{array}$ & $31-70$ & 11 & $118,9 \%$ \\
\hline \multirow[t]{2}{*}{ Vitamina B6 (mg/dia) } & $\begin{array}{c}1,7 \\
(0,17-3,82)\end{array}$ & $31-50$ & 1,1 & $154,5 \%$ \\
\hline & $\begin{array}{c}1,1 \\
(0,5-1,8)\end{array}$ & $51-70$ & 1,3 & $84,6 \%$ \\
\hline Vitamina B9 (mcg/dia) & $\begin{array}{c}181,03 \\
(20,5-54,5)\end{array}$ & $31-70$ & 320 & $56,5 \%$ \\
\hline Vitamina B12 (mcg/dia) & $\begin{array}{c}2,28 \\
(0,17-9,51)\end{array}$ & $31-70$ & 2,0 & $114 \%$ \\
\hline Vitamina C (mg/dia) & $\begin{array}{c}110,2 \\
95,65-14,4)\end{array}$ & $31-70$ & 60 & $183,6 \%$ \\
\hline Cálcio (mg/dia) & 459,4 & $31-70$ & 800 & $57,42 \%$ \\
\hline
\end{tabular}




\begin{tabular}{|c|c|c|c|c|}
\hline & $(85,29-2.000)$ & & & \\
\hline Fósforo (mg/dia) & $\begin{array}{c}843,46 \\
(169,3-2.108)\end{array}$ & $31-70$ & 580 & $145,4 \%$ \\
\hline \multirow[t]{2}{*}{ Ferro (mg/dia) } & $\begin{array}{c}9,13 \\
(1,68-21,82)\end{array}$ & $31-50$ & 8,1 & $112 \%$ \\
\hline & $\begin{array}{c}1,5 \\
(0,4-2,9)\end{array}$ & $51-70$ & 5 & $30 \%$ \\
\hline Zinco (mg/Dia) & $\begin{array}{c}6,76 \\
(0,78-25,1)\end{array}$ & $31-70$ & 6,8 & $99,4 \%$ \\
\hline Magnésio (mg/dia) & $\begin{array}{c}223,08 \\
(33,3-769,3)\end{array}$ & $31-70$ & 265 & $84,2 \%$ \\
\hline Selênio (mcg/dia) & $\begin{array}{c}69,4 \\
(8,25-312,34)\end{array}$ & $31-70$ & 45 & $154,2 \%$ \\
\hline Manganês (mg/dia) & $\begin{array}{c}1,8 \\
(0,17-7,28)\end{array}$ & $31-70$ & 1,8 & $100 \%$ \\
\hline \multirow[t]{2}{*}{ Sódio (mg/dia) } & $\begin{array}{c}1.000,7 \\
(69,27-9.911,8)\end{array}$ & & 1.500 & $66,6 \%$ \\
\hline & $\begin{array}{c}1400 \\
(800-2.800)\end{array}$ & $51-70$ & 1.300 & $107,6 \%$ \\
\hline Potássio (mg/dia) & $\begin{array}{c}2.161,24 \\
(452,7-7.778)\end{array}$ & $31-70$ & 4.700 & $45,9 \%$ \\
\hline
\end{tabular}

$\mathrm{EAR}=$ Estimated Average Requirement; $\mathrm{AI}=$ Adequate Intake. Fonte: Autores.

Tabela 7. Média e desvio padrão da ingestão de energia, macronutrientes, recomendações nutricionais e porcentagem de adequação em relação às recomendações nutricionais (DRIs), após análise do recordatório alimentar de 24 horas (R24h) de pacientes atendidos no ambulatório de nutrição da oncologia de um hospital público de Aracaju-SE.

\begin{tabular}{|c|c|c|c|c|}
\hline Variável & $\begin{array}{ll}\begin{array}{l}\text { Grupo } \\
\text { (anos) }\end{array} & \text { etário } \\
\end{array}$ & $\begin{array}{l}\mathrm{M} \pm \mathrm{DP} \text { (mínimo - } \\
\text { máximo) }\end{array}$ & $\begin{array}{l}\text { Recomendações } \\
\text { Nutricionais }\end{array}$ & $\begin{array}{l}\text { Porcentagem } \\
\text { adequação }\end{array}$ \\
\hline Calorias (Kcal/dia) & $31-70$ & $\begin{array}{l}1.415,5 \pm 651 \\
(231,9-4.492)\end{array}$ & $\begin{array}{ll}--- \\
-\end{array}$ & --- \\
\hline Carboidrato (\%) & $31-70$ & $\begin{array}{l}55,11 \pm 11,3 \\
(27,7-80,8)\end{array}$ & $45-65$ & 10 \\
\hline Proteína (\%) & $31-70$ & $\begin{array}{l}20,52 \pm 6,2 \\
(8,65-43,6)\end{array}$ & $10-35$ & 100 \\
\hline Lipídio (\%) & $31-70$ & $\begin{array}{l}24,9 \pm 9,4 \\
(8-59)\end{array}$ & $20-35$ & 100 \\
\hline Gordura monoinsaturada (\%) & $31-70$ & $\begin{array}{l}7,53 \pm 3,59 \\
(1,28-19,19)\end{array}$ & $\leq 20 \%$ & 100 \\
\hline Gordura Poli-insaturada (\%) & $31-70$ & $\begin{array}{l}4,28 \pm 1,96 \\
(1,01-10,1)\end{array}$ & $\leq 10 \%$ & 100 \\
\hline Gordura saturada $(\%)$ & $31-70$ & $\begin{array}{l}8,55 \pm 4,3 \\
(1,48-21,1)\end{array}$ & $<10 \%$ & 100 \\
\hline Colesterol (mg) & $31-70$ & $\begin{array}{l}284,9 \pm 212 \\
(1.265-43,7)\end{array}$ & $<300 \mathrm{mg}$ & 100 \\
\hline
\end{tabular}

Fonte: Autores.

Em uma associação entre alteração do peso e tipo de câncer, faixa etária, gênero e comorbidade (Tabela 8) 63 indivíduos apresentaram algum tipo de alteração no peso e 104 não apresentaram nenhum tipo de alteração, seja para ganho ou perda de peso. Pacientes com câncer de mama e cabeça e pescoço foram o que maior apresentaram casos de perda de peso $(\mathrm{n}=12)$, seguido dos de intestino e de útero e ovário $(\mathrm{n}=7)$. Os cânceres dos tipos: cabeça e pescoço, intestino, gástrico, hepático e outros tipos não apresentaram casos de ganho de peso. Em contraponto, pacientes avaliados que não apresentaram alterações 
no peso foram a maioria entre os casos, apenas o câncer de cabeça e pescoço apresentou um n maior para alteração no peso $(n=12)$ do que para não alteração $(n=7)$.

Tabela 8. Associação entre perda de peso e tipo de câncer, gênero, faixa etária e comorbidade de pacientes atendidos no ambulatório de nutrição da oncologia de um hospital público de Aracaju-SE

\begin{tabular}{|c|c|c|c|c|c|}
\hline \multicolumn{6}{|c|}{ Alteração de peso } \\
\hline & & Ganhou & Perdeu & Não mudou & $* \mathbf{p}$ \\
\hline \multirow[t]{9}{*}{ Tipo de Câncer } & Mama & 5 & 12 & 42 & \multirow{9}{*}{0,05} \\
\hline & Próstata & 1 & 2 & 7 & \\
\hline & Útero e ovário & 2 & 7 & 19 & \\
\hline & Intestino & 0 & 7 & 11 & \\
\hline & Cabeça e pescoço & 0 & 12 & 7 & \\
\hline & Hematológico & 3 & 4 & 4 & \\
\hline & Gástrico & 0 & 4 & 5 & \\
\hline & Hepático & 0 & 1 & 3 & \\
\hline & Outros & 0 & 3 & 6 & \\
\hline \multirow[t]{2}{*}{ Faixa etária } & Adulto & 9 & 36 & 70 & \multirow[b]{2}{*}{0,61} \\
\hline & Idoso & 2 & 16 & 34 & \\
\hline \multirow[t]{2}{*}{ Gênero } & Feminino & 9 & 39 & 76 & \multirow[b]{2}{*}{0,81} \\
\hline & Masculino & 2 & 13 & 28 & \\
\hline \multirow[t]{6}{*}{ Comorbidade } & Diabetes & 1 & 0 & 3 & \multirow{6}{*}{0,04} \\
\hline & Hipertensão arterial sistêmica & 0 & 6 & 14 & \\
\hline & Dislipidemia & 0 & 2 & 4 & \\
\hline & Mais de uma & 4 & 13 & 13 & \\
\hline & Outras doenças & 2 & 1 & 3 & \\
\hline & Não tem & 4 & 30 & 67 & \\
\hline
\end{tabular}

Teste Qui-quadrado com ${ }^{*} \mathrm{p} \leq 0,05$. Fonte: Autores.

Ainda em relação a tabela 8, caracterizando quanto a faixa etária, os adultos foram os que mais apresentaram alteração no peso (ganho n=9; perda: $n=36$ ) em relação aos idosos (ganho $n=2$; perda: $n=16$ ). Já relacionado à o gênero, as mulheres foram as que mais apresentaram alteração no peso (ganho $n=9$; perda: $n=39$ ) em relação aos homens (ganho $n=2$; perda: $n=13$ ). Do total de indivíduos que não sofreram alterações (n=104), 70 eram adultos e 34 idosos, desses, 76 eram mulheres e 28 homens.

Para as comorbidades avaliadas, Diabetes, hipertensão e dislipidemia os indivíduos que não apresentavam nenhuma dessas foram os que maior tiveram casos de alteração de peso $(n=34)$ sendo 30 casos de perda de peso e 4 de ganho. (Tabela 8 ).

A partir do estado nutricional em comparação com os tipos de tratamentos oncológicos avaliou-se que (Tabela 9): em relação ao IMC e os pacientes em quimioterapia, os que estavam acima do peso foram os que tiveram maior predominância $(\mathrm{n}=45)$ enquanto 30 foram avaliados em eutrofia. 
Tabela 9. Associação entre tipo de tratamento oncológico e estado nutricional de pacientes atendidos no ambulatório de nutrição da oncologia de um hospital público de Aracaju-SE

\begin{tabular}{|c|c|c|c|c|c|c|c|}
\hline & & $\mathbf{Q T}^{+}$ & $\mathbf{Q T}+\mathbf{R T}^{\boldsymbol{\beta}}$ & $\mathbf{R T}^{\mathbf{\omega}}$ & Não iniciou & Não Fez & $* \mathbf{p}$ \\
\hline \multirow[t]{3}{*}{$* * \mathbf{I M C}$} & Baixo peso & 15 & 8 & 0 & 1 & 0 & \\
\hline & Eutrófico & 30 & 10 & 4 & 3 & 2 & 0,23 \\
\hline & Acima do peso & 45 & 22 & 15 & 4 & 8 & \\
\hline \multirow[t]{6}{*}{ \#PCT } & Desnutrido Grave & 44 & 13 & 4 & 3 & 3 & \\
\hline & Desnutrido Moderado & 5 & 5 & 2 & 3 & 0 & \\
\hline & Desnutrido Leve & 12 & 9 & 2 & 0 & 3 & $\mathrm{O}, 009$ \\
\hline & Eutrófico & 14 & 8 & 1 & 0 & 2 & \\
\hline & Sobrepeso & 3 & 2 & 4 & 0 & 0 & \\
\hline & Obesidade & 12 & 3 & 6 & 2 & 2 & \\
\hline \multirow[t]{6}{*}{$\infty \mathbf{C B}$} & Desnutrido Grave & 12 & 9 & 1 & 2 & 1 & \\
\hline & Desnutrido Moderado & 26 & 7 & 3 & 2 & 3 & \\
\hline & Desnutrido Leve & 18 & 8 & 3 & 2 & 1 & 0,67 \\
\hline & Eutrófico & 17 & 13 & 7 & 0 & 3 & \\
\hline & Sobrepeso & 7 & 2 & 3 & 1 & 1 & \\
\hline & Obesidade & 10 & 1 & 2 & 1 & 1 & \\
\hline
\end{tabular}

Teste Qui-quadrado com *p $\leq 0,05 ; * *$ IMC $=$ índice de massa corporal; \#PCT = prega cutânea do tríceps; $\infty \mathrm{BC}=$ circunferência do braço. +quimioterapia, $\beta$ Quimioterapia+radioterapia, wradioterapia. Fonte: Autores.

O mesmo ocorreu nos pacientes em radioterapia $(n=15)$ e nos que realizavam ambos os tratamentos $(n=22)$, para os pacientes eutróficos o " $\mathrm{n}$ ” foi de 4 e 10 indivíduos respectivamente; tendo como referência o PCT os pacientes avaliados em desnutrição grave tiveram maior predominância nos que realizavam quimioterapia $(n=44)$. Em relação a CB os casos de eutrofia tiveram destaque nos que realizavam apenas radioterapia $(n=7)$ ou ambos os tratamentos $(n=13)$. Já para os que realizavam quimioterapia os casos de desnutrição grave foram mais predominantes $(n=26)$, enquanto para eutrofia foram identificados 17 indivíduos.

\section{Discussão}

No presente estudo, foi observado um déficit no consumo de vitamina A pelos pacientes. No estudo de Ferreira et al. (2016) os participantes apresentaram ingestão insuficiente de vitamina A e excesso de peso, podendo ser uma das variáveis que podem estar relacionados com o excesso de peso. Vários mecanismos foram propostos pelo qual a vitamina A e os carotenoides pré-formados podem ter efeitos anticarcinogênicos, devido ao seu papel no mecanismo de diferenciação celular anormal, principal característica do câncer, enquanto que a vitamina A é reconhecida por seu papel na regulação do crescimento, diferenciação e apoptose celular, o ácido retinóico demonstrou inibir a expressão de certos oncogenes in vitro e os carotenoides possuem função antioxidante e podem fornecer defesa contra as espécies reativas de oxigênio (EROs) que danificam o DNA (Hu et al., 2015).

A vitamina E é o principal antioxidante lipossolúvel presente nas membranas celulares, protegendo-as da peroxidação lipídica e eliminando radicais livres. Além disso, reduz o nitrito, inibindo a produção de nitrosoaminas e nitrosoamidas cancerígenas, potencializa a resposta imune, regula a agregação plaquetária e ativa a proteína quinase C22. A ingestão dietética adequada de vitamina E reduz o risco de câncer de mama em até $18 \%$ e sua deficiência severa está associada com um maior risco de câncer de mama (Bortoli et al., 2016). 
Estudos recentes têm demonstrado que a suplementação de vitamina D3 pode reduzir o risco de desenvolver câncer em adultos eutróficos sem diagnóstico da doença (Chandler et al., 2020). Uma revisão de Young e Xiong (2018) mostrou que a ingestão de altas doses de vitamina D (10.000UI/d) demonstrou possíveis efeitos antitumorais, inibindo o crescimento do câncer de mama.

A ingestão de vitamina B6 encontra-se reduzida, ela está envolvida em muitas reações bioquímicas e pode desempenhar um papel na carcinogênese. Um estudo em 2017 de 121 estudos observacionais (participantes $=1.924 .506$, casos de câncer $=$ 96.436) e 9 ensaios clínicos randomizados (RCTs) (participantes $=34.911$, casos $=2.539$ ) considerando 19 locais de tumor revelou que a alta ingestão de alimentos (apenas alimentos) a vitamina B6 foi significativamente associada com menor risco de alguns tipos de câncer (Mocellin et al., 2017).

A vitamina B9 é um nutriente essencial que ocorre naturalmente como folato, foi observado uma redução no consumo deste micronutriente. Uma análise conjunta de 23 estudos prospectivos envolvendo um total de 41.516 casos de câncer de mama e 1.171.048 indivíduos foram incluídos para meta-análise. Descobriu-se que a ingestão de folato está associada a uma redução do risco de desenvolver câncer de mama (Zeng et al., 2019).

A baixa ingestão de cálcio foi encontrada em nossa amostra, isto é um problema para indivíduos que tem câncer visando o tratamento da doença, Estudos tem evidenciado que a alimentação é um fator importante para o risco do desenvolvimento da doença, visto que cálcio e vitamina $\mathrm{D}$ são nutrientes que podem estar relacionados com a redução da gênese do câncer colorretal. Apesar disso, não há como concluir que o cálcio e a vitamina D podem fazer parte de uma intervenção visando a inibição do crescimento tumoral devido às limitações dos estudos (Cabral \& Gruezo, 2010).

Alguns tipos de câncer, como o de mama, podem ter o risco de mortalidade reduzido com o consumo adequado de vitamina $\mathrm{C}$ e uma das possibilidades desse efeito são as propriedades antioxidantes desse micronutriente, neutralizando os radicais livres (Harris et al., 2014). Yasueda et al. (2016) realizou um estudo utilizando ensaios clínicos randomizados controlados que analisaram a suplementação de suplementos antioxidantes em pacientes com qualquer tipo de câncer, durante o tratamento quimio e radioterápico. Foram analisados nutrientes como: vitaminas A, E, C, ubiquinona, zinco, selênio, entre outros. Não houve conclusões definitivas quanto à eficácia e segurança das vitaminas e minerais antioxidantes, sugerindo assim mais estudos para avaliar possíveis benefícios da suplementação.

Com relação à ingestão de macronutrientes, as pacientes apresentaram ingestão dentro do recomendado. Resultados semelhantes foram encontrados em Ferreira et al. (2016), porém Surwillo e Wawrzyniak (2013), observou deficiências nutricionais nas pacientes com câncer de mama quanto ao consumo calórico, ingestão de carboidratos e proteínas. Dessa forma, faz-se necessário uma alimentação balanceada para que o tratamento de pessoas com câncer obtenha mais eficácia.

Apesar dos resultados apresentados no presente estudo nos mostrar que os casos de não alteração de peso foram de maior predominância, há de se considerar que 38,4\% (n=63) em um n de 164 indivíduos, apresentaram algum tipo de alteração, tendo predominância maior para a perda de peso. Resultados parecidos foi encontrado no estudo de Brito et al. (2012) que analisou a perda de peso recente dos indivíduos através das ASGPPP (avaliação subjetiva global produzida pelo paciente). Os indivíduos perderam $10 \%$ ou mais do peso corporal, $42,6 \%$ dos indivíduos estavam em desnutrição leve/moderada e 15,8\% na faixa de desnutrição grave.

Essa perda de peso em pacientes com câncer é multifatorial, sendo a desnutrição a complicação mais frequentemente encontrada em pacientes oncológicos, a própria presença do tumor pode ser um dos fatores relacionados a essa perda de peso, induzindo à síndrome da anorexia-caquexia. Os tratamentos utilizados podem ser invasivos e com possíveis agravamentos do estado nutricional (Miranda et al., 2013).

Esse modelo de tratamento ainda causa muitos efeitos colaterais afetando células tumorais. Dentre outros efeitos colaterais estão: mielossupressão, vômitos, náuseas, diarreia e alopecia, com possíveis interferências no estado nutricional desses 
pacientes (Silva et al., 2012)

Os casos de perda de peso foram os de maior incidência em pacientes com câncer de cabeça e pescoço neste estudo. Segundo Pootz et al. (2020) tais casos apresentam um fato comum em resultados clínicos, a presença de marcadores inflamatórios em níveis elevados no sangue, como citocinas pró-inflamatórias, interleucina 1 (IL-1), interleucina 6 (IL-6) e fator de necrose tumoral alfa (TNF- $\alpha$ ), que aceleram as vias de catabolismo e dificultam a cicatrização. Isso promove intensa perda de massa muscular e tecido adiposo, gerando estado de resistência à insulina, inflamação sistêmica e ativação adrenérgica, o que pode levar o paciente à caquexia, gerando um estado de inflamação que resulta na diminuição da resposta ao tratamento, menor qualidade de vida e sobrevida, e maior tempo de permanência hospitalar.

Com relação ao ganho de peso, o resultado do estudo mostrou que o tipo de câncer que teve mais relevância foi o câncer de mama. Segundo Koo et al. (2016) os mecanismos sugeridos para ganho de peso pós-diagnóstico incluem diminuição da atividade física e alterações metabólicas associadas à quimioterapia. Vários estudos mostram que a quimioterapia está diretamente associada ao ganho de peso, como o estudo feito por Heideman et al. (2009) onde 271 pacientes diagnosticadas com câncer de mama na Holanda tiveram um aumento médio de $+2,4$ kg durante um tempo médio de acompanhamento de 3,5 anos.

As mudanças na composição corporal acontecem principalmente em mulheres e que realizam quimioterapia. Alterações na taxa metabólica basal, menopausa, protocolo que está sendo utilizado na quimioterapia, redução da atividade física e alimentação podem estar relacionados com as alterações corporais de indivíduos com câncer (Miranda et al., 2013).

Em outro estudo feito com 109 pacientes por Ginzac et al. (2020) chegou à conclusão que houve um impacto negativo da quimioterapia na atividade do tecido adiposo marrom, sendo esse um dos principais motivos que explicam o ganho de peso em mulheres com câncer de mama.

O tecido adiposo marrom é um órgão fascinante que possui um potencial termogênico muito grande por massa de tecido. Esse tecido tem uma capacidade surpreendente de mobilizar rapidamente seu próprio conteúdo de TG após a exposição ao frio. Os ácidos graxos dessa intensa lipólise intracelular são provavelmente os principais substratos para a termogênese do tecido adiposo marrom (Carpentier et al., 2018).

Com o tratamento a partir de quimioterapia e radioterapia, sintomas como vômitos, falta de apetite, diarreia, desconfortos abdominais, feridas e lesões na boca e na garganta que geram incômodos nos pacientes com câncer, repercutindo negativamente na ingestão alimentar e sendo uma das possíveis causas para a inadequação no consumo adequado de nutrientes e piorando o quadro clínico do paciente, visto que, há um aumento do gasto energético em pacientes com câncer (Ferreira et al., 2021; Freitas et al., 2020). Dessa forma, faz-se necessário a necessidade de um profissional nutricionista para minimizar a desnutrição desses pacientes devido a limitação para a ingestão alimentar.

\section{Conclusão}

As mulheres apresentaram maiores alterações no estado nutricional, com prevalência de sobrepeso e obesidade, inadequação na ingestão de micronutrientes, além da elevada perda de peso e maiores alterações em relação ao público masculino durante o tratamento. Dessa forma, faz-se necessário o acompanhamento nutricional com a finalidade de adequar a ingestão de nutrientes em pacientes oncológicos, evitando a perda de peso ou qualquer outra alteração referente ao estado nutricional, uma vez que ingestões inadequadas de nutrientes podem ser prejudiciais ao estado nutricional e a resposta ao tratamento, podendo ocasionar em uma reincidência do câncer.

\section{Referências}

Azevedo, C. D., \& Dal Bosco, S.M. (2011). Perfil nutricional, dietético e qualidade de vida de pacientes em tratamento quimioterápico. Conscientiae saúde, 10(1), 23-30. https://www.redalyc.org/pdf/929/92917188004.pdf 
Brasil, \& Conselho Nacional de Saúde. (2012). Resolução $\mathrm{n}^{\circ}$ 466/2012-Dispõe sobre pesquisa envolvendo seres humanos. https://conselho.saude.gov.br/resolucoes/2012/Reso466.pdf

Bortoli, M. C., Bandeira, V. S., \& Cozzolino, S. M. F. (2016). Vitamina E (tocoferol). In S.M.F. Cozzolino (Ed.), Biodisponibilidade de Nutrientes (5th ed.), 369-392. Manole.

Brito, L. F., Silva, L. S., Fernandes, D. D., Pires, R. A., Nogueira, A. D. R., Souza, C. L., \& Cardoso, L. G. V. (2012). Perfil nutricional de pacientes com câncer assistidos pela casa de acolhimento ao paciente oncológico do sudoeste da Bahia. Revista Brasileira de cancerologia,58(2), 163-171. https://doi.org/10.32635/2176-9745.RBC.2012v58n2.615

Cabral, C. M., \& Gruezo, N. D. (2010). Ingestão de cálcio e vitamina D e risco de câncer colorretal: uma revisão bibliográfica. Revista Brasileira de Cancerologia, 56(2), 259-266. https://doi.org/10.32635/2176-9745.RBC.2010v56n2.1506

Carpentier, A. C., Blondin, D. P., Virtanen, K. A., Richard, D., Haman, F. \& Turcotte, É. E. (2018). Brown Adipose Tissue Energy Metabolism in Humans. Frontiers in Endocrinology, 9, 447. https://doi.org/10.3389/fendo.2018.00447

Chandler, P. D., Chen, W. Y., Ajala, O. N., Hazra, A., Cook, N., Bubes, V., \& VITAL Research Group. (2020). Effect of vitamin D3 supplements on development of advanced cancer: A secondary analysis of the VITAL randomized clinical trial. JAMA network open, 3(11), e2025850-e2025850. https://doi.org/10.1001/jamanetworkopen.2020.25850

Chumlea, W. C., Guo, S., Roche, A. F., \& Steinbaugh, M. L. (1988). Prediction of body weight for the nonambulatory elderly from anthropometry. Journal of the American Dietetic Association, 88(5), 564-568. https://doi.org/10.1016/S0002-8223(21)02009-5

Chumlea, W. C., Guo, S. S., \& Steinbaugh, M. L. (1994). Prediction of stature from knee height for black and white adults and children with application to mobility-impaired or handicapped persons. Journal of the American Dietetic Association, 94(12), 1385-1391. https://doi.org/10.1016/0002-8223(94)92540-2

Ferreira, I. B., Marinho, E. D. C., Custódio, I. D. D., Gontijo, C. A., Paiva, C. E., Crispim, C. A., \& Maia, Y. C. D. P. (2016). Consumo alimentar e estado nutricional de mulheres em quimioterapia. Ciência \& Saúde Coletiva, 21, 2209-2218. https://doi.org/10.1590/1413-81232015217.05412015

Ferreira, N. P., Pardo, J. de A., \& Salomon, A. L. R. (2021). Declínio do estado nutricional infanto-juvenil durante o tratamento oncológico: seus agravantes e a relevância da terapia nutricional. Research, Society and Development, 10(16), e481101624229. https://doi.org/10.33448/rsd-v10i16.24229

Freitas, C. B. de, Veloso, T. C. P., Segundo, L. P. da S., Sousa, F. P. G. de, Galvão, B. S., \& Paixão, P. A. R. (2020). Prevalência de desnutrição em pacientes oncológicos. Research, Society and Development, 9(4), e192943019. https://doi.org/10.33448/rsd-v9i4.3019

Frisancho, A. R. (1990). Anthropometric standards for the assessment of growth and nutritional status. University of Michigan press.

Ginzac, A., Barres, B., Chanchou, M., Gadéa, E., Molnar, I., Merlin, C., Coudert, B., Thivat, E. \& Durando, X. (2020). A decrease in brown adipose tissue activity is associated with weight gain during chemotherapy in early breast cancer patients. BMC Cancer 2020 20:1, 20(1), 1-7. https://doi.org/10.1186/s12885020-6591-3

Gozzo, T. de O., Souza, S. G. de, Moysés, A. M. B., Carvalho, R. A. de O. de \& Ferreira, S. M. de A. (2015). Conhecimento da equipe de enfermagem acerca de eventos adversos do tratamento quimioterápico. Ciência, Cuidado e Saúde, 14(2), 1058-1066. https://doi.org/10.4025/cienccuidsaude.v14i2.25040

Harris, H. R., Orsini, N. \& Wolk, A. (2014). Vitamin C and survival among women with breast cancer: a meta-analysis. European Journal of Cancer, 50(7), 1223-1231. https://doi.org/10.1016/j.ejca.2014.02.013

Heideman, W.H., Russell, N.S., Gundy, C., Rookus, M.A. \& Voskuil, D.W. (2009). The frequency, magnitude and timing of post-diagnosis body weight gain in Dutch breast cancer survivors. European Journal of Cancer, 45(1), 119-126. https://doi.org/10.1016/j.ejca.2008.09.003

Hu, F., Wu, Z., Li, G., Teng, C., Liu, Y., Wang, F., Zhao, Y. \& Pang, D. (2015). The plasma level of retinol, vitamins A, C and $\alpha$-tocopherol could reduce breast cancer risk? A meta-analysis and meta-regression. Journal of Cancer Research and Clinical Oncology, 141(4), 601-614. https://doi.org/10.1007/s00432-014$1852-7$

INCA. (2020). Brasil terá 625 mil novos casos de câncer a cada ano do triênio 2020-2022. https://www.inca.gov.br/noticias/brasil-tera-625-mil-novos-casos-decancer-cada-ano-do-trienio-2020-2022

Kanda, M. H., Contim, D., Gonçalves, J. R. L. \& Santos, É. A. (2014). A Percepção Dos Familiares Cuidadores Sobre O Tratamento Quimioterápico Em Crianças E Adolescentes. Cogitare Enfermagem, 19(1), 84-88. https://www.redalyc.org/pdf/4836/483647660012.pdf

Koo, H.Y., Seo, Y.G., Cho, M.H., Kim, M.J. \& Choi, H.C. (2016). Weight Change and Associated Factors in Long-Term Breast Cancer Survivors. PLoS ONE, 11(7). https://doi.org/10.1371/journal.pone.0159098

Kuczmarski, M. F., Kuczmarski, R. J., \& Najjar, M. (2000). Descriptive anthropometric reference data for older Americans. Journal of the American Dietetic Association, 100(1), 59-66. https://doi.org/10.1016/S0002-8223(00)00021-3

Lipschitz, D. A. (1994). Screening for nutritional status in the elderly. Primary Care: Clinics in Office Practice, 21(1), 55-67. https://doi.org/10.1016/S00954543(21)00452-8

Miranda, T. V., Neves, F. M. G., Costa, G. N. R., \& de Souza, M. A. M. (2013). Estado nutricional e qualidade de vida de pacientes em tratamento quimioterápico. Revista Brasileira de Cancerologia, 59(1), 57-64. https://doi.org/10.32635/2176-9745.RBC.2013v59n1.544

Mocellin, S., Briarava, M. \& Pilati, P. (2017). Vitamin B6 and Cancer Risk: A Field Synopsis and Meta-Analysis. Journal of the National Cancer Institute, 109(3), 1-9. https://doi.org/10.1093/jnci/djw230

Padovani, R. M., Amaya-Farfán, J., Colugnati, F. A. B., \& Domene, S. M. Á. (2006). Dietary reference intakes: aplicabilidade das tabelas em estudos nutricionais. Revista de Nutrição, 19, 741-760. https://doi.org/10.1590/S1415-52732006000600010 
Research, Society and Development, v. 11, n. 1, e51111125142, 2022

(CC BY 4.0) | ISSN 2525-3409 | DOI: http://dx.doi.org/10.33448/rsd-v11i1.25142

Poltronieri, T. S. \& Tusset, C. (2016). Impacto Do Tratamento Do Câncer Sobre O Estado Nutricional De Pacientes Oncológicos: Atualização Da Literatura. Revista Brasileira de Ciências Da Saúde, 20(4), 327-332. https://doi.org/10.4034/RBCS.2016.20.04.10

Pootz, S. C., Boff, D. G., Canuto, R., Brollo, J. \& Silva, A. C. P. da. (2020). Aconselhamento Nutricional em Pacientes com Câncer de Cabeça, Pescoço e Esôfago em Tratamento (Quimio)Radioterápico. Revista Brasileira de Cancerologia, 665(1), e-13531. https://doi.org/10.32635/2176-9745.RBC.2020v66n1.531

Port G.Z. (2012). Avaliação nutricional bioquímica de pacientes portadores de cirrose com carcinoma hepatocelular. Dissertação de mestrado, Universidade Federal de Ciências da Saúde de Porto Alegre, Porto Alegre, RS, Brasil.

Silva, A. M., De, L., Franco, P., Serafim, T., Santos, S., Passos, X. S. \& Ferreira Costa, B. M. (2012). Impacto das aversões alimentares no estado nutricional de pacientes oncológicos submetidos à quimioterapia. J Health Sci Inst, 30(2), 166-170. https://repositorio.unip.br/wpcontent/uploads/2020/12/V30_n2_2012_p166-170.pdf

Souza, R. G. De, Lopes, T. do V. C., Pereira, S. S., Soares, L. P. \& Pena, G. das G. (2017). Avaliação do estado nutricional, consumo alimentar e capacidade funcional em pacientes oncológicos. Braz J Oncol, 13(44), 1-11. https://cdn.publisher.gn1.link/brazilianjournalofoncology.com.br/pdf/v13n44a04.pdf

Surwillo, A., \& Wawrzyniak, A. (2013). Nutritional assessment of selected patients with cancer. Rocz Panstw Zakl Hig, 64(3), 225-233. http://wydawnictwa.pzh.gov.pl/roczniki_pzh/download-article?id=990

World Health Organization. (1998). Obesity: preventing and managing the global epidemic: report of a WHO consultation on obesity, Geneva, 3-5 June 1997 (No. WHO/NUT/NCD/98.1). World Health Organization. https://apps.who.int/iris/bitstream/handle/10665/63854/WHO_NUT_NCD_98.1_\%28p1158\%29.pdf?sequence=1\&isAllowed=y

Yasueda, A., Urushima, H. \& Ito, T. (2016). Efficacy and Interaction of Antioxidant Supplements as Adjuvant Therapy in Cancer Treatment: A Systematic Review. Integrative Cancer Therapies, 15(1), 17-39. https://doi.org/10.1177\%2F1534735415610427

Young, M. R. I., \& Xiong, Y. (2018). Influence of vitamin D on cancer risk and treatment: Why the variability?. Trends in cancer research, 13, 43-53. https://www.ncbi.nlm.nih.gov/pmc/articles/PMC6201256/pdf/nihms-988517.pdf

Zeng, J., Wang, K., Ye, F., Lei, L., Zhou, Y., Chen, J., Zhao, G. \& Chang, H. (2019). Folate intake and the risk of breast cancer: an up-to-date meta-analysis of prospective studies. European Journal of Clinical Nutrition, 73(12), 1657-1660. https://doi.org/10.1038/s41430-019-0394-0 